hep-ph/0205268

$\mathrm{IC} / 2002 / 34$

$\mathrm{SINP} / \mathrm{TNP} / 02-18$

\title{
Gauge unification in 5-D $S U(5)$ model with orbifold breaking of GUT symmetry
}

\author{
Biswajoy Brahmachari ${ }^{a, c}$ 用 and Amitava Raychaudhuri ${ }^{b, c}$ 间 \\ (a) Theoretical Physics Group, Saha Institute of Nuclear Physics \\ AF/1 Bidhannagar, Kolkata 700064, India \\ (b) Department of Physics, University of Calcutta \\ 92, Acharya Prafulla Chandra Road, Kolkata 700009, India \\ (c) Abdus Salam International Centre for Theoretical Physics \\ Strada Costiera 11, 34014 Trieste, Italy
}

\begin{abstract}
$\underline{\text { ABSTRACT }}$
We consider a 5-dimensional $S U(5)$ model wherein the symmetry is broken to the 4-dimensional Standard Model by compactification of the 5th dimension on an $S^{1} /\left(Z_{2} \times Z_{2}^{\prime}\right)$ orbifold. We identify the members of all $S U(5)$ representations upto $\mathbf{7 5}$ which have zero modes. We examine how these light scalars affect gauge coupling unification assuming a single intermediate scale and present several acceptable solutions. The 5-D compactification scale coincides with the unification scale of gauge couplings and is determined via this renormalization group analysis. When $S O(10)$ is considered as the GUT group there are only two solutions, so long as a few low dimensional scalar multiplets upto $\mathbf{1 2 6}$ are included.
\end{abstract}

\footnotetext{
*e-mail: biswajoy@theory.saha.ernet.in
}

†e-mail: amitava@cubmb.ernet.in 


\section{Introduction}

The $S U(5)$ model[1] unifies the strong, weak, and electromagnetic interactions in the smallest simple group. It has many other attractive features which are well recognized. But it suffers from the following two major difficulties which are actually generic to the idea of grand unification[2] itself. (i) Because quarks and leptons reside in unified multiplets and there are B- and L-violating interactions, gauge boson exchanges can result in proton decay [3]. If these gauge bosons are appropriately heavy, the decay rate will be very small. Their masses, in the usual formulation, are, however, not arbitrary but rather determined by the scale where the different gauge couplings unify. The proton decay lifetime is therefore a robust prediction of the model. No experimental signature of proton decay [4] has been found yet and the model is disfavoured. More complicated unification models involving several intermediate mass-scales can evade this problem[5]. (ii) The low energy Higgs doublet, responsible for electroweak breaking, is embedded in a 5 representation of $S U(5)$. The other members of this multiplet are color triplet scalars which must have a mass near the unification scale - since no such scalars have been observed at the electroweak scale. This leads to an unnatural mass splitting among the members of the same $S U(5)$ multiplet. This is termed the double-triplet splitting problem [6].

These two unwelcome features of the $S U(5)$ model can be tackled in an elegant way if unified $S U(5)$ symmetry exists in a 5-D world. Low energy 4D $S U(3)_{c} \times S U(2)_{L} \times U(1)_{Y}$ symmetry is recovered when the extra dimension is compactified on a $S^{1} /\left(Z_{2} \times Z_{2}^{\prime}\right)$ orbifold [7]. This situation is realized when space-time is considered to be factorized into a product of $4 \mathrm{D}$ Minkowski space-time $M^{4}$ and the orbifold $S^{1} /\left(Z_{2} \times Z_{2}^{\prime}\right)$. The coordinate system consists of $x^{\mu}=\left(x^{0}, x^{1}, x^{2}, x^{3}\right)$ and $y=x^{5}$. There are two distinct 4 -D branes; one at $y=0$ and another at $y=\pi R / 2$. On the $S^{1}, y=0$ is identified with $y=\pi R\left(Z_{2}^{\prime}\right.$ symmetry) while $y= \pm \pi R / 2$ are identified with each other $\left(Z_{2}\right.$ symmetry).

As is common in models of this type, we assume that the fermions are located in the 4 -D brane at $y=0$ while the gauge bosons and the scalars are allowed to travel in the bulk. The discrete $Z_{2}$ and $Z_{2}^{\prime}$ symmetries, which we refer to as $P$ and $P^{\prime}$, permit the expansion of any 5 -D field $\phi$ in the following mode 
expansions according to whether they are even or odd under $\left(P, P^{\prime}\right)$ :

$$
\begin{array}{rlrl}
\phi_{++}(y)= & \sqrt{\frac{2}{\pi R}} \sum_{n=0}^{\infty} \phi_{++}^{(2 n)} \cos \frac{2 n y}{R} ; & & M_{n}=\frac{2 n}{R} \\
\phi_{-+}(y)=\sqrt{\frac{2}{\pi R}} \sum_{n=0}^{\infty} \phi_{-+}^{(2 n+1)} \sin \frac{(2 n+1) y}{R} ; & M_{n}=\frac{2 n+1}{R} \\
\phi_{+-}(y)=\sqrt{\frac{2}{\pi R}} \sum_{n=0}^{\infty} \phi_{+-}^{(2 n+1)} \cos \frac{(2 n+1) y}{R} ; & M_{n}=\frac{2 n+1}{R} \\
\phi_{--}(y)=\sqrt{\frac{2}{\pi R}} \sum_{n=0}^{\infty} \phi_{--}^{(2 n+2)} \sin \frac{(2 n+2) y}{R} ; & M_{n}=\frac{2 n+2}{R}
\end{array}
$$

Here $n=0,1,2, \ldots$ and we have suppressed the $x^{\mu}$ dependence. The behaviour of the fields under $P$ and $P^{\prime}$ can be read off from the subscripts in the left hand side above. For example, $\phi_{-+}$is odd under $P$ and even under $P^{\prime}$. We have also listed the masses of the different modes. Notice that only the $\phi_{++}$field can have a massless mode. One of the prime motivations of these higher dimensional $S U(5)$ models is to ensure doublet-triplet splitting within the 5 scalar multiplet of $S U(5)$ and to ensure that from within the adjoint representation $(\mathbf{2 4})$ of the gauge bosons only the $S U(3)_{c} \times S U(2)_{L} \times U(1)_{Y}$ gauge bosons remain massless. Both can be achieved by ascribing $P, P^{\prime}$ parities of ++ to the $(1,2,1 / 2)$ submultiplet in the $\mathbf{5}$ while the remaining $(3,1,-1 / 3)$ states carries +- parity composition of the $S U(5)$ representations upto 75 are listed in Table (11) [0]. Since $P, P^{\prime}$ commute with the Standard Model (SM) gauge symmetry $S U(3)_{c} \times S U(2)_{L} \times U(1)_{Y}$, the $Z_{2} \times Z_{2}^{\prime}$ parities for the members of the higher $S U(5)$ multiplets can be built up from this assignment for the scalars in the fundamental representation?. These parities have been indicated in Table (1). In this way we can also assure that the $(1,1,1)+(1,3,0)+(8,1,0)$ multiplets of 24 remain massless, breaking $S U(5)$ symmetry below the compactification scale $1 / R \equiv M_{X}$.

Above $M_{X}$, the mass scale of the non-SM gauge bosons, $X, Y$, and that of the colour triplet scalars in the $\mathbf{5}$ representation, $S U(5)$ symmetry is unbroken. This scale is determined in our analysis by the unification of the three SM gauge couplings. We assume one intermediate scale, $M_{I}$, such that all scalars which are permitted to have a zero mode, excepting the SM scalar doublet,

\footnotetext{
${ }^{1}$ Here we are using the decomposition of the $S U(5)$ multiplets under $S U(3)_{c} \times S U(2)_{L} \times$ $U(1)_{Y}$.

${ }^{2}$ In principle, one can assign arbitrary $Z_{2} \times Z_{2}^{\prime}$ parities to the submultiplets of the higher $\mathrm{SU}(5)$ representations. However, to reduce adhocness, here we work with the ansatz that once we assign the parities for the fundamental representation, those for the submultiplets of higher $S U(5)$ representations are determined by group theoretic relationships.
} 


\begin{tabular}{|c|c|}
\hline$S U(5) \supset$ & $S U(3)_{c} \times S U(2)_{L} \times U(1)_{Y}$ \\
\hline $5 \supset$ & $(1,2,1 / 2)_{++}+(3,1,-1 / 3)_{+-}$ \\
\hline$\overline{5} \supset$ & $(1,2,-1 / 2)_{+}++(\overline{3}, 1,1 / 3)_{+-}$ \\
\hline $10 \supset$ & $(1,1,1)_{++}+(\overline{3}, 1,-2 / 3)_{+}++(3,2,1 / 6)_{+}$ \\
\hline $15 \supset$ & $(1,3,1)_{++}+(3,2,1 / 6)_{+-}+(6,1,-2 / 3)_{+}+$ \\
\hline $24 \supset$ & $(1,1,0)_{++}+(1,3,0)_{++}+(3,2,-5 / 6)_{+-}+(\overline{3}, 2,5 / 6)_{+-}+(8,1,0)_{++}$ \\
\hline $35 \supset$ & $(1,4,-3 / 2)_{++}+(\overline{3}, 3,-2 / 3)_{+-}+(\overline{6}, 2,1 / 6)_{++}+(\overline{10}, 1,1)_{+-}$ \\
\hline $40 \supset$ & $\begin{array}{l}(1,2,-3 / 2)_{+}++(3,2,1 / 6)_{+}++(\overline{3}, 1,-2 / 3)_{+-}+(\overline{3}, 3,-2 / 3)_{+}- \\
+(8,1,1)_{+-}+(\overline{6}, 2,1 / 6)_{+}+\end{array}$ \\
\hline $45 \supset$ & $\begin{array}{l}(1,2,1 / 2)_{+}+(3,1,-1 / 3)_{+}+(3,3,-1 / 3)_{+}++(\overline{3}, 1,4 / 3)_{+}- \\
+(\overline{3}, 2,-7 / 6)_{+}++(\overline{6}, 1,-1 / 3)_{+}+(8,2,1 / 2)_{+}+\end{array}$ \\
\hline $50 \supset$ & $\begin{array}{l}(1,1,-2)_{+}++(3,1,-1 / 3)_{+}++(\overline{3}, 2,-7 / 6)_{+-}+(\overline{6}, 3,-1 / 3)_{+}- \\
+(6,1,4 / 3)_{++}+(8,2,1 / 2)_{+-}\end{array}$ \\
\hline $70 \supset$ & $\begin{array}{l}(1,2,1 / 2)_{+}++(1,4,1 / 2)_{++}+(3,1,-1 / 3)_{+}-+(3,3,-1 / 3)_{+}- \\
+(\overline{3}, 3,4 / 3)_{+-}+(6,2,-7 / 6)_{+}++(8,2,1 / 2)_{+}+(15,1,-1 / 3)_{+-}\end{array}$ \\
\hline $70^{\prime} \supset$ & $\begin{array}{l}(1,5,-2)_{++}+(\overline{3}, 4,-7 / 6)_{+-}+(\overline{6}, 3,-1 / 3)_{+}++(\overline{10}, 2,1 / 2)_{+}- \\
+(\overline{15}, 1,4 / 3)_{++}\end{array}$ \\
\hline $75 \supset$ & $\begin{array}{l}(1,1,0)_{+}+(3,1,5 / 3)_{+}+(3,2,-5 / 6)_{+-}+(\overline{3}, 1,5 / 3)_{+}+ \\
+(\overline{3}, 2,5 / 6)_{+-}+(\overline{6}, 2,-5 / 6)_{+-}+(6,2,5 / 6)_{+-}+(8,1,0)_{+}+ \\
+(8,3,0)_{+}\end{array}$ \\
\hline
\end{tabular}

Table 1: The $S U(3)_{c} \times S U(2)_{L} \times U(1)_{Y}$ contents of the different $S U(5)$ representations. Also shown are the $P$ and $P^{\prime}$ assignments.

pick up a mass at this scale. We include their contributions to the beta functions of the one loop renormalization group equations (RGE) and solve for both the intermediate scale $M_{I}$ and the unification scale $M_{X}$. The beta function coefficients are given by:

$$
b_{i}=\left(\begin{array}{c}
0 \\
-22 / 3 \\
-11
\end{array}\right)+n_{f}\left(\begin{array}{l}
4 / 3 \\
4 / 3 \\
4 / 3
\end{array}\right)+T_{s}^{i} / 3
$$

We take $n_{f}=3$. The above expression assumes that the scalar fields are complex. For real scalar fields one has to use $T_{s}^{i} / 6$ in Eqn. (11). The $T_{s}^{i}$ 


\begin{tabular}{|ccccc|}
\hline $\mathrm{R}$ & light scalar multiplets & $T_{s}^{3}$ & $T_{s}^{2}$ & $T_{s}^{1}$ \\
\hline $\mathbf{5}$ & $(1,2,1 / 2)$ & 0 & $1 / 2$ & $3 / 10$ \\
$\mathbf{1 0}$ & $(1,1,1)+(\overline{3}, 1,-2 / 3)$ & $1 / 2$ & 0 & $7 / 5$ \\
$\mathbf{1 5}$ & $(1,3,1)+(6,1,-2 / 3)$ & $5 / 2$ & 2 & $17 / 5$ \\
$\mathbf{2 4}$ & $(1,3,0)+(8,1,0)$ & 3 & 2 & 0 \\
$\mathbf{3 5}$ & $(1,4,-3 / 2)+(\overline{6}, 2,1 / 6)$ & 5 & 8 & $28 / 5$ \\
$\mathbf{4 0}$ & $(1,2,-3 / 2)+(3,2,1 / 6)+(\overline{6}, 2,1 / 6)$ & 6 & 5 & 3 \\
$\mathbf{4 5}$ & $(1,2,1 / 2)+(\overline{3}, 2,-7 / 6)+(8,2,1 / 2)$ & 7 & 6 & $38 / 5$ \\
$\mathbf{5 0}$ & $(1,1,-2)+(3,1,-1 / 3)+(\overline{6}, 3,-1 / 3)+(6,1,4 / 3)$ & $21 / 2$ & 3 & $51 / 5$ \\
$\mathbf{7 0}$ & $(1,2,1 / 2)+(1,4,1 / 2)+(6,2,-7 / 6)+(8,2,1 / 2)$ & 11 & $25 / 2$ & $131 / 10$ \\
$\mathbf{7 0}$ & $(1,5,-2)+(\overline{6}, 3,-1 / 3)+(\overline{15}, 1,4 / 3)$ & 25 & 22 & $146 / 5$ \\
$\mathbf{7 5}$ & $(3,1,5 / 3)+(\overline{3}, 1,-5 / 3)+(8,1,0)+(8,3,0)$ & 13 & 16 & 10 \\
\hline
\end{tabular}

Table 2: The contributions to the $\beta$-functions from the light members of the different $S U(5)$ representations upto $\mathbf{7 5}$.

for the light scalar submultiplets of the different $S U(5)$ representations upto 75 are listed in Table (2). Defining $m_{k, l}=\ln \left(m_{k} / m_{l}\right)$ and $b_{k, l}^{i}$ to be the $\beta$ coefficients governing evolution in the range $m_{k} \leftrightarrow m_{l}$, we get the following three solutions of the RGE.

$$
2 \pi \alpha_{i}^{-1}\left(M_{Z}\right)=2 \pi \alpha_{X}^{-1}+b_{X, I}^{i} m_{X, I}+b_{I, Z}^{i} m_{I, Z}
$$

Using the values of couplings at the low energy scale $M_{Z}$

$$
\alpha_{1}\left(M_{Z}\right)=0.01688, \quad \alpha_{2}\left(M_{Z}\right)=0.03322, \quad \alpha_{3}\left(M_{Z}\right)=0.117
$$

we solve the three equations in Eqn. (2). First, we present a simple illustrative example below.

\section{Simple example}

Because the GUT symmetry is broken via orbifolding, let us consider the case where there are only $\mathbf{5}$-plets of $S U(5)$ Higgs scalars at the unification scale and assume that there are $n_{5}$ of them. Then compactification allows only doublets to be light and not their triplet partners. In this case the $\beta$ 
coefficients are given by,

$$
b_{X, I}^{i}=\left(\begin{array}{c}
41 / 10 \\
-19 / 6 \\
-7
\end{array}\right)+\frac{n_{5}}{3}\left(\begin{array}{c}
3 / 10 \\
1 / 2 \\
0
\end{array}\right)
$$

Solving Eqn. (2) we obtain

$$
\alpha_{X}^{-1}=38.53, m_{I, Z}=26.98-194.75 / n_{5}, m_{X, I}=194.75 / n_{5}
$$

Because $m_{I, Z} \geq 0$ we obtain $n_{5} \geq 8$. For the case of $n_{5}=8$ we get,

$$
M_{I}=1.39 \mathrm{TeV}, M_{X}=5.0 \times 10^{10} \mathrm{TeV} .
$$

The GUT scale $M_{X}$ is rather low but it is consistent with proton decay because of the existence of $Z_{2} \times Z_{2}^{\prime}$ parity. Note that the intermediate scale is in a very attractive region phenomenologically. Eight Higgs doublets can be degenerate at this scale of 1.3-1.4 TeV. They may play an important role in the fermion mass puzzle. Further, the scale $M_{X} \simeq 10^{10} \mathrm{TeV}$ is interesting from the point of view of the see-saw mechanism. The unification pattern is shown in Fig. (11).

\section{More general cases}

We now turn to the more general possibility where scalars in higher representations of $S U(5)$ are present. The light scalars of all $S U(5)$ multiplets upto 75 and their contributions to the beta coefficients are listed in Table (2).

\subsection{Small number of representations and low interme- diate scales}

We consider upto the $\mathbf{7 5}$ dimensional representation of $S U(5)$ and demand that the threshold scale, $M_{I}$, be less than $10 \mathrm{TeV}$. For the sake of economy, we also consider only those solutions where for every representation $R$, the number $n_{R}$ is either 0 or 1 . If we do not put any restriction on the number of representations, but maintain that $n_{R}$ be zero or unity only, then we get 43 different solutions. In Table (3) we list those solutions for which not more than two $n_{R}$ are non-zero. 


\begin{tabular}{|ccc|}
\hline $\begin{array}{c}\text { Representations } \\
\text { with } n_{R}=1\end{array}$ & $\begin{array}{c}M_{I} \\
(\mathrm{TeV})\end{array}$ & $\begin{array}{c}M_{X} \\
(\mathrm{TeV})\end{array}$ \\
\hline $\mathbf{3 5}$ & 0.223 & $1.61 \times 10^{11}$ \\
$\mathbf{5 , 3 5}$ & 5.70 & $1.38 \times 10^{11}$ \\
$\mathbf{2 4 , 3 5}$ & 0.905 & $5.50 \times 10^{11}$ \\
$\mathbf{3 5 , 4 0}$ & 3.61 & $1.84 \times 10^{12}$ \\
$\mathbf{4 5 , 7 5}$ & 3.61 & $1.84 \times 10^{11}$ \\
\hline
\end{tabular}

Table 3: $S U(5)$ repesentations of scalars whose light members ensure coupling constant unification. The unification scale, $M_{X}$, and the intermediate scale, $M_{I}$, are also given.

Though the intermediate scales, $M_{I}$, and the unification scales, $M_{X}$, in the last two cases are the same, the value of $\alpha_{X}$ turns out to be 0.037 and 0.710 , respectively.

Let us explain one case in more detail. Let there be only 35-plets of $S U(5)$ at the unification scale and assume that there are $n_{35}$ of them. Then compactification allows only $(1,4,-3 / 2)+(\overline{6}, 2,1 / 6)$ fields at low energy. In this case the $\beta_{i}$ coefficients are given by,

$$
b_{X, I}^{i}=\left(\begin{array}{c}
41 / 10 \\
-19 / 6 \\
-7
\end{array}\right)+\frac{n_{35}}{3}\left(\begin{array}{c}
28 / 5 \\
8 \\
5
\end{array}\right)
$$

Solving the RGE we obtain

$$
\alpha_{X}^{-1}=32.72, m_{I, Z}=28.20-27.3073 / n_{35}, m_{X, I}=27.3073 / n_{35}
$$

Because $m_{I, Z} \geq 0$ we obtain $n_{35} \geq 1$. For the case of $n_{35}=1$ we get,

$$
M_{I}=0.223 \mathrm{TeV}, M_{X}=1.6 \times 10^{11} \mathrm{TeV} .
$$

\subsection{Low dimensional representations only}

Another alternative which we examine is by restricting to $S U(5)$ representations upto 24 subject further to the requirements $n_{5}<8, n_{10}<5, n_{15}<$ 
$5, n_{24}<5$. Then we get the results given in Table (44) when we impose $M_{I}<70 \mathrm{TeV}$. The unification patterns of the gauge couplings for a few sample cases are shown in Fig. (1).

\begin{tabular}{|cccccc|}
\hline$n_{5}$ & $n_{10}$ & $n_{15}$ & $n_{24}$ & $M_{I}(\mathrm{TeV})$ & $M_{X}(\mathrm{TeV})$ \\
\hline 8 & 0 & 0 & 0 & 1.678 & $4.66 \times 10^{10}$ \\
7 & 0 & 0 & 1 & 0.223 & $1.61 \times 10^{11}$ \\
8 & 0 & 0 & 1 & 5.696 & $1.38 \times 10^{11}$ \\
7 & 0 & 0 & 2 & 0.905 & $5.49 \times 10^{11}$ \\
8 & 0 & 0 & 2 & 19.08 & $4.05 \times 10^{11}$ \\
7 & 0 & 0 & 3 & 3.607 & $1.84 \times 10^{12}$ \\
8 & 0 & 0 & 3 & 63.08 & $1.17 \times 10^{12}$ \\
6 & 0 & 0 & 4 & 0.407 & $1.33 \times 10^{13}$ \\
7 & 0 & 0 & 4 & 14.12 & $6.08 \times 10^{12}$ \\
6 & 0 & 0 & 5 & 0.199 & $5.22 \times 10^{13}$ \\
7 & 0 & 0 & 5 & 54.36 & $1.97 \times 10^{13}$ \\
8 & 1 & 0 & 5 & 0.407 & $1.33 \times 10^{13}$ \\
8 & 0 & 1 & 5 & 0.407 & $1.33 \times 10^{13}$ \\
\hline
\end{tabular}

Table 4: Various $S U(5)$ scrnarios which gives low intermediate scales upto $70 \mathrm{TeV}$

\section{Remarks about $S O(10)$}

It might be of interest to extend this analysis to grand unification groups of higher rank. It is readily seen that the solutions will become more difficult to come by. For example, we give in Table (国) the $S U(5)$ contents of the $S O(10)$ representations upto $\mathbf{1 2 6}$. Notice, that the inclusion of a single $\mathbf{1 2 6}$ of $S O(10)$ is equivalent to the simultaneous presence of $\overline{\mathbf{5}}, \mathbf{1 0}, \overline{\mathbf{1 5}}, \mathbf{4 5}, \overline{\mathbf{5 0}}$ repesentations of $S U(5)$ and there is no flexibility of including the $S U(5)$ representations individually. 


\begin{tabular}{|ccccc|}
\hline $\mathrm{R}$ & $\mathrm{SU}(5)$ components & $T_{s}^{3}$ & $T_{s}^{2}$ & $T_{s}^{1}$ \\
\hline $\mathbf{1 0}$ & $\mathbf{5}+\overline{\mathbf{5}}$ & 0 & 1 & $3 / 5$ \\
$\mathbf{1 6}$ & $\mathbf{1}+\overline{\mathbf{5}}+\mathbf{1 0}$ & $1 / 2$ & $1 / 2$ & $17 / 10$ \\
$\mathbf{4 5}$ & $\mathbf{1}+\mathbf{1 0}+\overline{\mathbf{1 0}}+\mathbf{2 4}$ & 4 & 2 & $14 / 5$ \\
$\mathbf{1 2 0}$ & $\mathbf{5}+\overline{\mathbf{5}}+\mathbf{1 0}+\overline{\mathbf{1 0}}+\mathbf{4 5}+\overline{\mathbf{4 5}}$ & 15 & 13 & $93 / 5$ \\
$\mathbf{1 2 6}$ & $\mathbf{1}+\overline{\mathbf{5}}+\mathbf{1 0}+\overline{\mathbf{1 5}}+\mathbf{4 5}+\overline{\mathbf{5 0}}$ & $41 / 2$ & $23 / 2$ & $229 / 10$ \\
\hline
\end{tabular}

Table 5: The contributions to the $\beta$-functions from the light members of the different $\mathrm{SO}(10)$ representations upto $\mathbf{1 2 6}$.

If we permit all $\mathrm{SO}(10)$ representations upto $\mathbf{1 2 6}$ and consider no more than upto 8 of any single representation then we find just two allowed solutions:

1) $n_{10}=8$, other $n_{i}=0 \Rightarrow M_{I}=1.68 \mathrm{TeV}, M_{X}=4.67 \times 10^{10} \mathrm{TeV}$

2) $n_{10}=6, n_{16}=1$, other $n_{i}=0 \Rightarrow M_{I}=295 \mathrm{TeV}, M_{X}=7.99 \times 10^{9} \mathrm{TeV}$

\section{Conclusions and Discussion}

In this work, we have examined the light scalar modes that survive when a 5-dimensional $S U(5)$ model reduces to the 4-dimensional SM through the orbifold compactification route. The scalars which are permitted to have zero modes are assumed to pick up a mass at some scale $M_{I}$ intermediate between the electroweak and Planck scales. They contribute to the beta coefficients in the $M_{I}<\mu<M_{X}$ regime. The compactification scale, $M_{X}$, above which $S U(5)$ is unbroken, is determined by the unification of the gauge couplings. This analysis also determines $M_{I}$. We identify solutions for which $M_{I}$ is in an interesting phenomenological range and can be probed at the next generation colliders. This analysis is somewhat similar in spirit to the approach chosen for supersymmetric-GUTs where the SUSY scale is fixed by gauge unification.

It is seen from Table (2) that $P$ is $(+)$ for all the multiplets. Thus it does not play any role in the present analysis. However, we would like to keep the option of generalizing this method to the supersymmetric case where $P$ has a non-trivial role. 
The scale of degenerate scalars, $M_{I}$, should be treated as an approximate one in the sense that in reality some spread in the masses around it can be expected. The standard model doublet has a mass at the electroweak scale. This should not be viewed as an unnatural fine tuning as some relevant Yukawa couplings can be of order $10^{-2}-10^{-3}$.

When the unification symmetry is assumed to be of higher rank, then the number of acceptable solutions reduces dramatically. For $S O(10)$ just two solutions can be obtained, so long as we stick to the low dimensional representations of the symmetry group.

At first sight it might seem that we are introducing too many scalar degrees of freedom. However, this appears less dramatic when we compare it to the Minimal Supersymmetric Standard Model where too a large number of scalars are required.

Acknowledgements: This work was done while both authors were visiting the Abdus Salam International Centre for Theoretical Physics, Italy. They are grateful to the High Energy Section and the Associateship Office of the Centre for hospitality. The research of AR is supported by CSIR, India. 


\section{References}

[1] H. Georgi, S.L. Glashow, Phys. Rev. Lett. 32438 (1974); H. Georgi, H. R. Quinn, S. Weinberg, Phys. Rev. Lett. 33, 451 (1974).

[2] J. C. Pati, A. Salam, Phys. Rev. D10, 275 (1974); Jogesh C. Pati, A. Salam, Phys. Rev. D8, 1240 (1973); R. N. Mohapatra, J. C. Pati, Phys. Rev. D11, 2558 (1975); F. Gursey, P. Ramond, P. Sikivie, Phys. Lett. B60, 177 (1976)

[3] A.J. Buras, J. R. Ellis, M.K. Gaillard, D.V. Nanopoulos, Nucl. Phys. B135, 66 (1978); C. Jarlskog, Phys. Lett. B82, 401 (1979); C. Jarlskog, F.J. Yndurain, Nucl. Phys. B149, 29 (1979); F. Wilczek, A. Zee, Phys. Rev. Lett. 43, 1571 (1979); M. Machacek, Nucl. Phys. B159, 37 (1979)

[4] SuperKamiokande Collaboration (Y. Hayato et al.), Phys. Rev. Lett. 831529 (1999); Super-Kamiokande Collaboration (M. Shiozawa et al.), Phys. Rev. Lett. 813319 (1998); W. Gajewski et al., Phys.Rev. D42, 2974 (1990); Soudan-2 Collaboration (W.W.M. Allison et al.), Phys. Lett. B427 217 (1998).

[5] P. H. Frampton, B. Hoon Lee, Phys. Rev. Lett. 64619 (1990); P. H. Frampton, T. W. Kephart, Phys. Rev. D42, 3892 (1990); B. Brahmachari, U. Sarkar, R. B. Mann, T. G. Steele, Phys. Rev. D45, 2467 (1992); B. Brahmachari, U. Sarkar, Phys. Lett. B303, 260 (1993); P. B. Pal, Phys. Rev. D45, 2566 (1992); B. Brahmachari, Phys. Rev. D48, 1266 (1993)

[6] H. Georgi, Phys. Lett. 108B, 283 (1982); B. Grinstein, Nucl. Phys. B206, 387 (1982); A. Masiero, D.V. Nanopoulos, K. Tamvakis, T. Yanagida, Phys. Lett. B115 380 (1982); S. Dimopoulos, F. Wilczek, NSF-ITP-82-07 (unpublished); A. Sen, Phys. Rev. Lett. 55, 33 (1985);

[7] Y. Kawamura, Prog. Theor. Phys. 103, 613 (2000); Y. Kawamura, Prog. Theor. Phys. 105, 691 (2001); G. Altarelli, F. Feruglio, Phys. Lett. B511, 257 (2001); A. Hebecker, J. March-Russell, Nucl. Phys. B613, 3 (2001); J. A. Bagger, F. Feruglio, F. Zwirner, Phys. Rev. Lett. 88 101601 (2002); Y. Nomura, D. R. Smith, N. Weiner, Nucl. Phys. B613, $147(2001)$ 


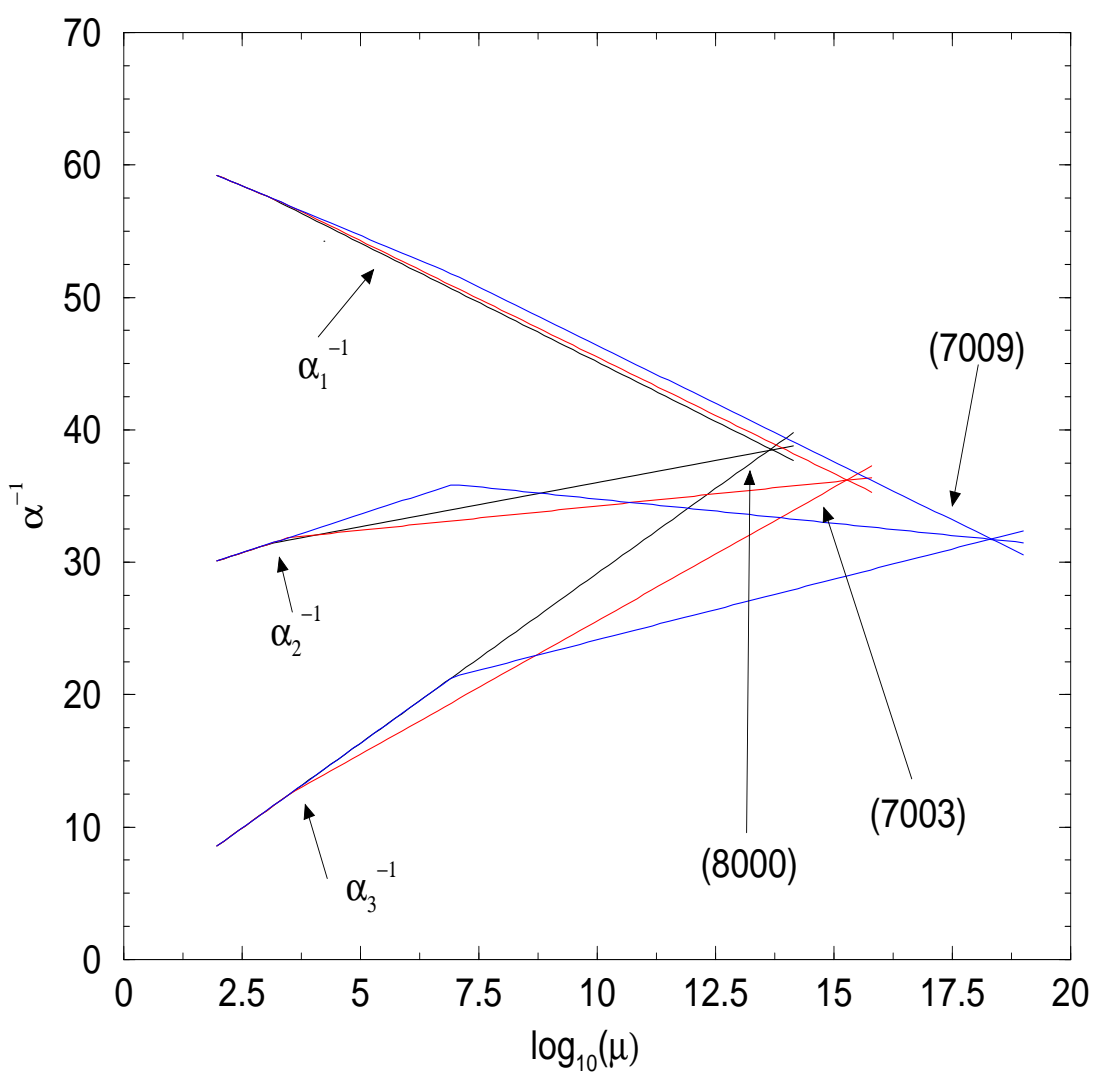

Figure 1: Gauge unification in various models. Labels of cases are $\left(n_{5}, n_{10}, n_{15}, n_{24}\right)$. As a first approximation we have used one intermediate scale which is given by the mass scale of extra scalars allowed by $S^{1} / Z_{2} \times Z_{2}^{\prime}$ compactifications. 